\title{
Letter to the Editor Regarding Comparative Efficacy of JAK Inhibitors for Moderate-to-Severe Rheumatoid Arthritis: A Network Meta-Analysis
}

\author{
Walid Fakhouri (D) - Christophe Sapin • Thorsten Holzkaemper • \\ Inmaculada de la Torre
}

Received: September 9, 2020 / Accepted: January 29, 2021 / Published online: March 20, 2021

(c) The Author(s) 2021

\section{Key Summary Points}

The authors of the network meta-analysis did not address the issue of heterogeneity between the studies resulting from differences in baseline patient characteristics.

There were inappropriate inclusion criteria of trials, with lack of adjustment for differences in key treatment effect modifiers such as prior biologic disease modifying anti-rheumatic drugs (DMARDs) use and methotrexate dose at baseline. In addition, there was no assessment of the impact of the differences in the number of conventional synthetic DMARDs failures allowed between studies.

There was lack of adjustment for placebo arm response across trials.

These major limitations of the analysis make the findings, and consequently the conclusions, inaccurate and therefore biased.

W. Fakhouri $(\bowtie) \cdot$ C. Sapin · T. Holzkaemper . I. de la Torre

Eli Lilly \& Company, Indianapolis, IN, USA e-mail: Fakhouri_walid@lilly.com

\section{LETTER}

Dear Editor,

We read the manuscript authored by Pope et al. (2020) on "Comparative Efficacy of JAK Inhibitors for Moderate-To-Severe Rheumatoid Arthritis: A Network Meta-Analysis" with great interest.

The network meta-analysis (NMA) found that upadacitinib $15 \mathrm{mg}$ once daily had numerically higher efficacy in terms of American College of Rheumatology (ACR) response and clinical remission among approved Janus kinase (JAK) inhibitor combination therapies and monotherapies for patients with inadequate response to conventional synthetic DMARD (csDMARD) treatments. While the authors acknowledged the limitations of their analyses, there is uncertainty that, if they had appropriately addressed limitations that may have been able to be evaluated, the conclusions of these analyses would have remained the same.

While focusing on phase III randomized controlled trials may partially address the issue of heterogeneity resulting from differences in trial design, it does not address the issue of heterogeneity between the studies resulting from major differences in baseline patient characteristics. Most notably, the authors did 
not address differences in the trials related to key treatment effect modifiers.

Firstly, the authors' networks included baricitinib trials where patients enrolled in the trials had no prior biologic DMARD (bDMARD) use and upadacitinib and tofacitinib trials where enrolled patients had prior bDMARD use $(\leq 20 \%)$. We believe this approach is not appropriate for primary analyses, as different previous exposure to bDMARD might constitute a source of bias. A more methodologically sound approach would have been either to only include baricitinib trials in a sensitivity analysis or to investigate the influence of prior biologic exposure through meta-regression techniques [1].

Secondly, differences between trials related to the number of patients with csDMARD failures between studies should have been mentioned and addressed. For example, upadacitinib SELECT-NEXT trial [2] allowed a maximum of two csDMARD failures while baricitinib RA-BUILD trial [3] had no restriction on the previous number of csDMARDs (no maximum numbers).

Thirdly, inclusion of trials allowing for lower background dose of methotrexate (MTX) in the network might also constitute another source of heterogeneity, the impact of which on treatment response has not been reported. We specifically refer here to the inclusion of RABALANCE [4] and SELECT-SUNRISE [5], in some of the networks, where the dosage of MTX was much lower than that reported in other included trials in the networks. Trials including Asians-Japanese patients with lower MTX dose should have been excluded in a sensitivity analysis and impact on results compared.

While the authors acknowledge that differences in placebo arm response rates across trials could be a potential confounder of the estimated treatment, they did not adjust the analyses for these differences. This is an issue that might have an impact on the results. Various statistical techniques exist (including adjustment on baseline risk) that could have been used to control for the differences in placebo response rate across trials. An assessment is needed, and missing in this NMA, on how differences in placebo rate response associated with differences in treatment outcomes.

As such, we believe the reported NMA has key limitations, some of which could have been addressed in the analyses, for the results to be valid. While the authors acknowledged some of the limitations, these were not properly addressed with their methodology. We also believe "numerically better results" in an NMA are very inconclusive results to be used for clinical relevance extrapolation and should be avoided as part of the key conclusions.

\section{DIGITAL FEATURES}

This article is published with digital features to facilitate understanding of the article. To view digital features for this article go to https://doi. org/10.6084/m9.figshare.13655846.

\section{ACKNOWLEDGEMENTS}

Funding. No Rapid Service Fee was received by the journal for the publication of this article.

Authorship. All named authors meet the International Committee of Medical Journal Editors (ICMJE) criteria for authorship for this article, take responsibility for the integrity of the work as a whole, and have given their approval for this version to be published.

Disclosures. Walid Fakhouri, Christophe Sapin, Thorsten Holzkaemper, and Inmaculada de la Torre, declare that they are employees and shareholders of Eli Lilly \& Company Ltd.

Compliance with Ethics Guidelines. This article is based on previously conducted studies and does not contain any studies with human participants or animals performed by any of the authors.

Open Access. This article is licensed under a Creative Commons Attribution-NonCommercial 4.0 International License, which permits any non-commercial use, sharing, adaptation, 
distribution and reproduction in any medium or format, as long as you give appropriate credit to the original author(s) and the source, provide a link to the Creative Commons licence, and indicate if changes were made. The images or other third party material in this article are included in the article's Creative Commons licence, unless indicated otherwise in a credit line to the material. If material is not included in the article's Creative Commons licence and your intended use is not permitted by statutory regulation or exceeds the permitted use, you will need to obtain permission directly from the copyright holder. To view a copy of this licence, visit http://creativecommons.org/licenses/bync/4.0/.

\section{REFERENCES}

1. Dias S, Sutton AJ, Welton NJ, Ades AE. NICE DSU technical support document 3: heterogeneity: subgroups, meta-regression, bias and bias-adjustment. 2011; last updated April 2012; available from http://www.nicedsu.org.uk.

2. Burmester GR, Kremer JM, van den Bosch F, et al. Safety and efficacy of upadacitinib in patients rheumatoid arthritis and inadequate response to conventional synthetic disease-modifying anti-rheumatic drugs (SELECT-NEXT): a randomized, doubleblind, placebo-controlled phase 3 trial. Lancet. 2018;391:2503-12.

3. Dougados $M$, van der Heijde D, Chen $Y$, et al. Baricitinib in patients with inadequate response or intolerance to conventional synthetic DMARDs: results from the RA-BUILD study. Ann Rheum Dis. 2017;76:88-95.

4. Li Z, Hu J, Bao C, et al. Efficacy and safety of baricitinib in mtx-ir patients with rheumatoid arthritis: 52 week results from a phase 3 study (RABALANCE). Ann Rheum Dis. 2018;77:969-70.

5. Kameda H, Takeuchi T, Yamaoka K, et al. Efficacy and safety of upadacitinib in Japanese patients with rheumatoid arthritis (SELECT-SUNRISE): a placebocontrolled phase IIb/III study. Rheumatology. 2020;59:1-11. 